\title{
BREAKING THE SILENCE: THE USE OF COLOR BARS
}

\author{
Jermaine V. Beltran \\ University of the Philippines Baguio, Philippines
}

\begin{abstract}
Test patterns or "color bars" as used by engineers in the television broadcasting industry to check the quality of colors a TV station in broadcasting has evolved in usage and meaning online. In 2017, Facebook timelines of TV5 Network, Inc.'s current and former employees were inundated with color bars. The color bars became signifiers and symbols of varied emotions as a visual graphic in the online space. Survey and interview of current and laid-off employees of TV5 were utilized to describe the evolving meaning of transcendence of emotions as displayed by visual graphics in the online space. Raising the questions: 1) Are "color bars" the new symbol of freedom of expression that connote to struggle and resistance against oppressive broadcasting industries in the online space?, 2) Are these "colors bars," as displayed demonstrates assertion of rights in the offline space?, and, 3) If this visual graphic is the new form of resistance, is the broadcasting industry in the Philippines listening? Findings showed that "color bars" were used as a form of protest of employees to show their emotional state when they learned that they were laid off from the company. The respondents inadvertently presented these "color bars" as signs of grief and solidarity in the online space to display resistance against such company decision. This represented the power resistance and visual communication on meaning expectation and resolutions between and among employees and media organizations.
\end{abstract}

Keywords: Mass media, organizational communication, power resistance

\section{Introduction}

The internet has truly revolutionized how humans have communicated in recent years. It offers a plethora of tools providing new space to interact with others. As such, social media has become one of the platforms in the internet that have made a difference because it provides an unlimited space where worlds,(literally and figuratively), collide. The online space, specifically that of social media platforms enables the merging of text and visuals through images, graphics, video, and audio as messages. Through these message elements, social media has afforded a place to react, share, and comment in topics which they can relate to. Popularity in the use of social media sites are not only because of the merging of texts and visuals, but it expands its usage in communication from ordinary social interactions to that of information sharing, creating a bevy of communication exchanges in one online platform.

Though social media can utilize text for social exchange online, visuals are much more favored by many as a way to substitute for thoughts. Aside from virally captivating videos, a lot of people utilize simple static images or graphics to convey a message in the social media. Thus, news feeds of users are inundated with various content; at times, too varied that it becomes a "hodge podge" of information.

In the last week of February 2017, a certain group of users in Facebook uploaded an interesting image that saturated many news feeds of their friend connections through the posting or displaying of an image of rainbow of colors which is not often seen by many. These rainbow of colors, otherwise known as color bars is used as a test pattern by engineers in the broadcast industry to check on the accuracy and quality of color transmission of their television station or network. Seeing the color bars in the online space, which is often seen in television, prompted such study. 
Color bars on television has different kinds of patterns, but what was displayed in the social media site, are color bars used by The Society of Motion Picture and Television Engineers (SMPTE) for test patterns standard. (Please see Image 1 below).

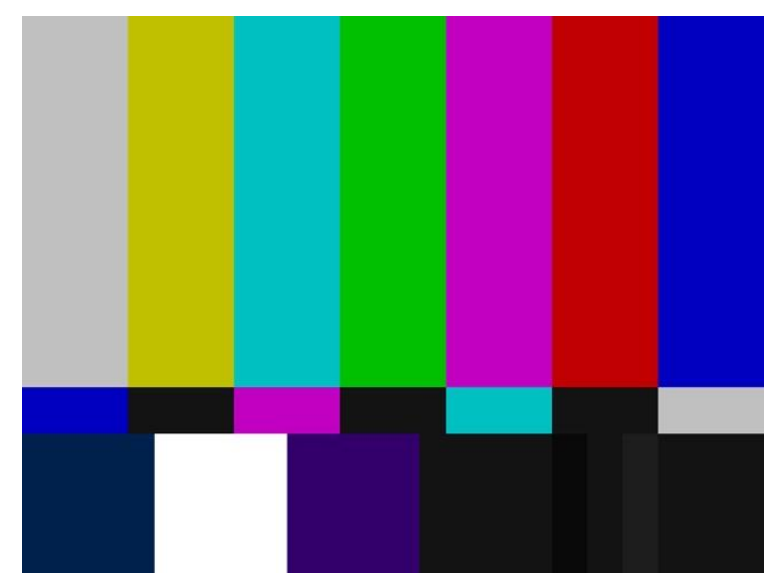

Image 1. A sample of SMPTE color bars

The SMPTE color bars, were posted by a number of former employees who were laid-off by TV5 Network, Inc., in their timelines from February 24 to February 28, 2017.

Ranking third in AGB Nielsen and Kantar audience ratings, TV5 Network, Inc. is a broadcast network in the Philippines, that has earned the reputation as one of the influential television networks in the country. Amidst this reputation, in February 24, 2017, TV5 Network, Inc. management decided to do a massive lay-off of 98 employees (supervisors, managers and rank and file employees). Reasons for this is because the employees were rendered as unfit for their positions, and, to reduce operational costs (Rappler.com, 2017). Counter-arguing this mass lay-off, employees' union of TV5 said that such move was abrupt and unjust. Moreover, employees' union of TV5 explains that this is an ironic of the network's vision for news casting that take parlance for the protection of the basic rights of disempowered sectors in their reportage but violates simple rules of labor contract of their own employees.

This occurrence has lead the researcher to wonder on the how and why the phenomenon transpired. Thus, this study is aimed at answering the following questions:

1. Why did the respondents choosecolor bars over other images to present their message?

2. How were the "color bars" used in the online space as a sign of assertion of rights?

3. How effective was the use of the color bars as a symbol of resistance against oppressive organizational cultures?

\section{Method}

The researcher used a mixed-method research design. The qualitative data were taken from a purposive sample. Social media posts from Facebook were gathered from February 24 to 28, 2018 specifically posts containing color bars, regardless if there is a caption or none. If there was a text or description, textual analysis was utilized to determine its connection to the image.The contents and technical use of the image itself was no longer included in the analysis since the study is seen through the lens of social media and the users who are in the middle of the issue at hand. The aforementioned period was the specific duration when the massive lay-off in the company happened.

After determining the affected employees who posted in Facebook, the researcher proceeded to gather quantitative data through an online questionnaire sent to 20 concerned employees. However, only 10 responded. They were specifically chosen since they are affected parties in this issue and are rank-and-file employees. The questionnaires were then subjected to coding analysis to check on the trends of their answers. 
In order to answer the questions stated in this study, the researcher primarily employed Charles Peirces Sign Theory (Semiosis)to analyze the motives of the respondents on the use of the color bars, while Chad Brinsfield's Organizational Silence was utilized to understand the problem between the officials of the company, its employees, and the ensuing result of this interaction.

The Repurposing of the denotation of the "Color Bars"

Based on the responses from those who posted the color bars, 9 out of 10 used this type of image since it is the most "convenient" way of presenting their sentiments about the issue. Considering that most of those who responded were cameramen and assistant cameramen, they said the color bars were used in their standard operating procedure in shooting and editing videos. The protocol was to record color bars in the beginning and end of a certain coverage in the news gathering process in the field. This was to properly separate raw, unedited videos to facilitate easy post-production editing and archiving. This is akin to chapters in a book or subheads in novels or written texts.

The original use of color bars as a behind-the-scene aspect of the broadcast production process transcended to the public sphere through social media. As mentioned earlier, since most were cameramen, they used a familiar image which they used at work. The image was used as an irony since this was analogous to the end of their video shoot but also the end of their careers in the company (Screenshot 1).

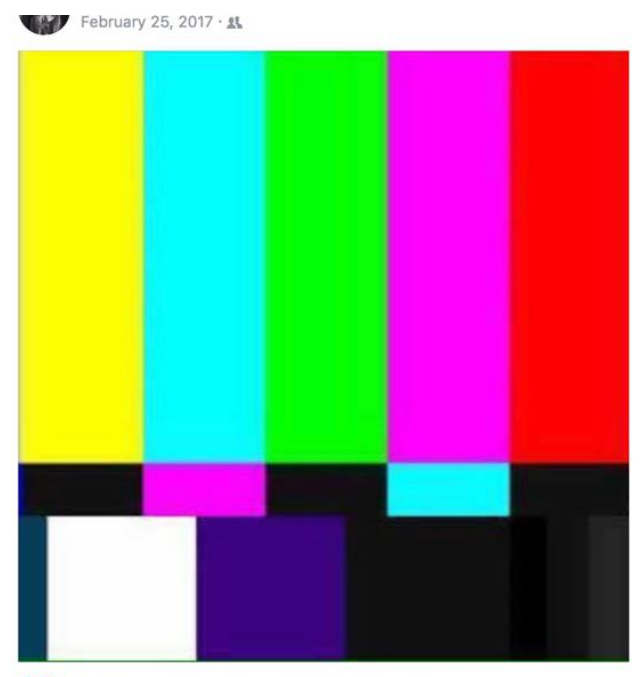

00 Carlito A. Balmaceda, Jerry Tabadero and 15 others

Screenshot 1. An example of a post from a former cameraman

Another employee went to the point of replacing his profile picture to color bars (Screenshot 2). He said the color bars as a profile picture was a bold way to represent what has transpired. This in effect, showed how personal the effect this has been to him. The usual profile picture is used to see the face of the user, however, he chose to replace it as a representation of personal distress. The color bars was once again repurposed not only as an ordinary post but a personal artifact of life for this particular user. In addition, the technical image, in effect, became a personal image in the process. 


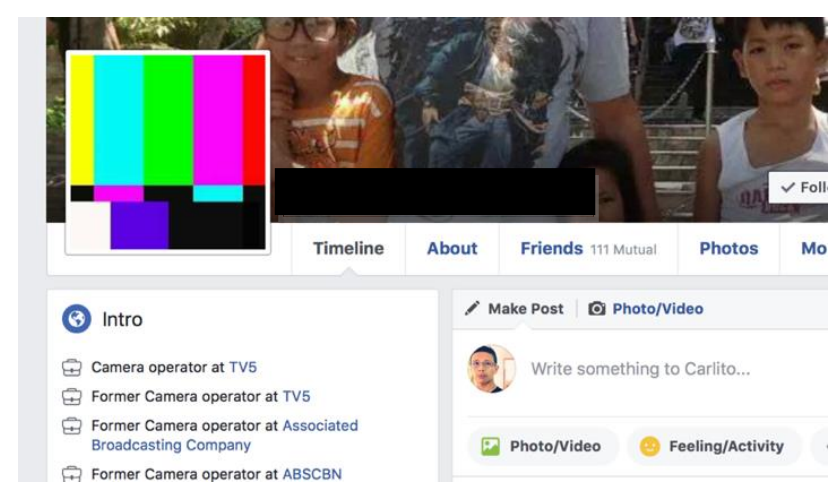

Screenshot 2. An assistant cameraman changed his profile picture to color bars

In this case, Pierce's Semiosis (Atkin, 2013)may explain the phenomena. The represent a men or signifier is the image "color bars". Its definition or referent (what the sign stands for)was changed from having a technical definition to a different meaning which is dependent on the user's motives on what to convey to his/her audiences. However, at this stage, the interpretant/signified has not yet been determined as the behavior of posting the color bars is an action, without the need for thinking yet of those who are going to comprehend the image.

In addition, the color bars were preferred since it was viewed as "neutral", basing on the original denotation as a test pattern with no related connotation of opinion or subjectivity. The "neutral" nature of the image lent to its ease or convenience as it does not immediately get judgement or sentiment from their audience in social media. This explains why many of these employees chose to use the image over text.

Some of the respondents, however, posted with descriptions but these were limited to a few words or phrases. A few of their words were:

- "Signing-off" / "Temporary signing-off"

- "The final countdown..."

- "Sayang ang pagod, puyat at pagsisikap" (Such a waste of effort, sleepless nights, and hard work)

Even these descriptions are neutral in nature as well, but it still implies about the sentiment of the user. Nevertheless, it directly relates to their standard that the image is used at the end of a video shoot correlating to their termination from the job.

Other than those who were affected by the lay-off, some indirect actors in this phenomenon have emerged. One such actor was aformer reporter who resigned from his post 1 year before this event. He said he posted the image because it was previously used by those who posted earlier in their timelines when the lay-off started.(See screenshot 3).As a contrast from those who were laid off, his post contained a description or caption about the event wherein he voiced his support and admiration to those who were terminated from their work. Although not directly talking about color bars in the caption, his use of the color bars was repurposed as a sign of support to those affected by the issue. 


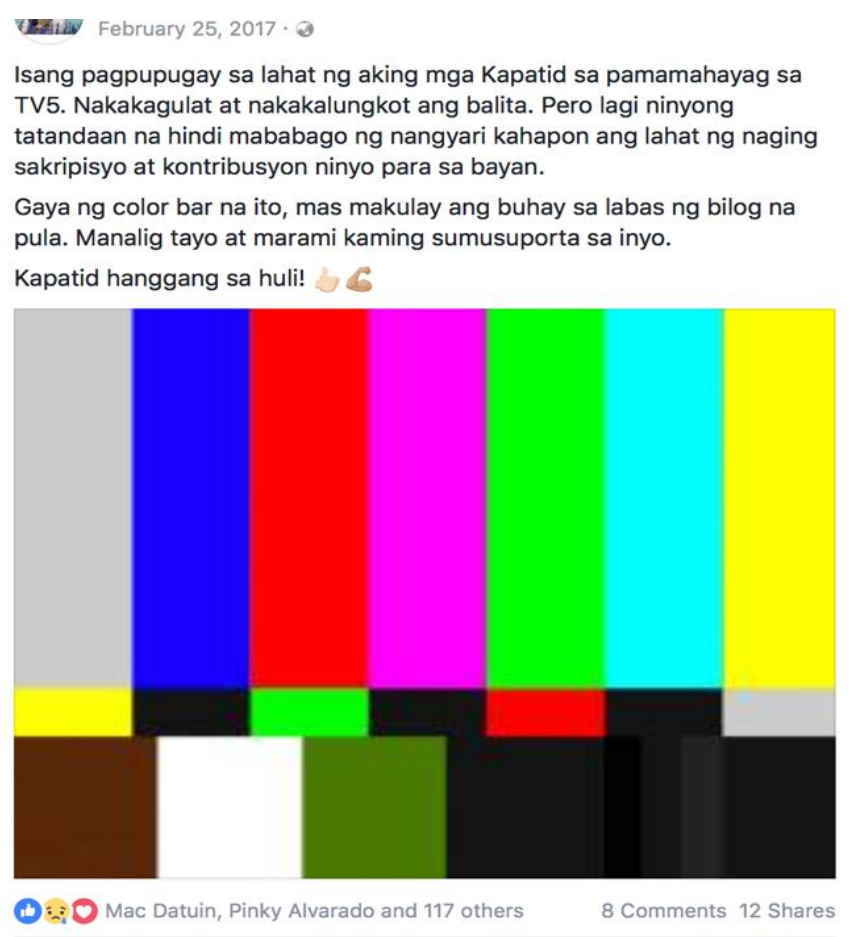

Screenshot 3. A former reporter sharing the same type of color bars but with corresponding description

Employees who were laid off were not the only ones who posted and repurposed the color bars. Former reporters and writers who already left the company followed suit in posting the color bars in their social media account, either as a post, profile picture, or cover photo, to symbolize unity and empathy with their former colleagues.Some resorted to sharing other user's posts and reposting it in their timelines. Despite the same image being used, it was repurposed by different users through captions or descriptions showing support. It may seem like the image was used to call attention to a certain problem, than just an aesthetic aspect of their posts, but it has unintentionally created an advocacy to help those affected by the retrenchment. This mindset for advocacy also added to the referent of the said image from only sentiments to a certain form of support or activism from those indirectly involved in this event. It then

\section{The Color Bars as a virtual sign of resistance}

According to the respondents, all of them were unjustly terminated. They expressed disappointment and dismay on the procedures that the management undertook to fulfill the retrenchment. The emotional burden was a factor in leading them to post the image. Albeit not technically connected to what the original meaning, the color bars were their sign of protest in the online space. Most of them mentioned the following reasons in their move to "virtually" protest:

- Absence of clear focus or direction for the organization and for making major decisions

- Difficulty with multiple personalities in the organization

- Failure of the company to train or retrain employees for professional growth

- Poor communication and feedback amongst people in the organization

- Shortage of funds for general operations

- Conflict amongst management and rank-and-file employees

- Lack of understanding of costs and operations of various resources in the company

- Low morale 
- Poor planning, organizing, leading, and administration of resources

- Suggestions are not accepted, or the management dismisses them

- Lack of transparency of the company's finances

- Management is "out of reach" or does not consult employees

Looking closely, their sentiments brought about their problems with the management and supervisors of the company. One main theme here is the staunch stance of the management not to budge or adjust to the needs of the rank-and-file employees. As seen in some of the screenshots (See Screenshot 4), the color bars were presented but there were no accompanying caption or description to what was the purpose of posting such image. This is a manifestation of what some researchers call as "organizational silence". Devised by Chad Brinsfield, "organizational silence" is separated by voice and silence itself. Voice is defined as "the expression of ideas, information, opinions, or concerns while silence can be defined as withholding those." (Begheri, et.al., 2012, pp. 49). In this effect, Pinder and Harlos (2001) defined silence "as the absence of voice as it has its own form of communication, involving a range of cognitions, emotions, or intentions" (ibid). In most cases, silence is established through the organizational structure and beliefs system of a company's supervisors and higher management to the lower-ranking employees.

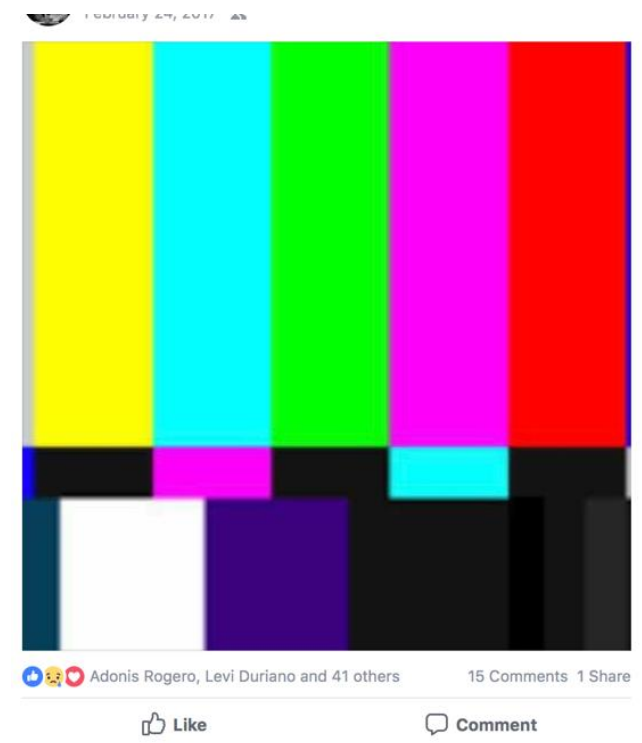

Screenshot 4. A sample of a post from a former assistant cameraman containing only color bars

In the case of TV5, concerns from employees (voice) are not explicitly expressed in the online space. This is ironic since there is no written code about the use of social media accounts of their employees which give them virtually total freedom from posting, especially in Facebook. However, the corporate structure of the organization lends to the somewhat "muted" state of these employees. Nevertheless, they had to use an image to represent their opinions or sentiments in the issue in which they are the main affected parties in this move of the managements. As a result, the image transcended their work and personal lives to the public sphere through their Facebook accounts. It is important to note that all of the respondents were rank-and-file employees who are in the lower ranks of the organization. This action also revealed the culture of the organization on how it treats its employees especially in dissent or unfavorable opinion. Although the network has no set guidelines or rule book about social media posts, the corporate culture in the company has lent to this phenomenon.

The actions seen in Facebook is a way for the employees, particularly those who were retrenched, to break the culture of "silence" in the organization. The silence can be observed in the rather "safe" uncontroversial nature of the use of color bars and lack or non-inclusion of a caption to the image. If there are descriptions or captions, there was no mention of the name of the company but only their involvement to their work in general. This shows a certain fear from the users of possible retaliation from their superiors. Although not explicitly seen in 
their posts, this behavior shows the company's clout on the employees despite having no "standards" set by the company on the use of social media. Thus, the freedom afforded by social media, Facebook in this case, has given them the avenue to present their voices to the public which they cannot truly accomplish in the company. Interestingly, almost all of the respondents said their objective on posting was not only to let the public know their sentiments and problems in the company but to let their own company know and understand their predicament. It is apparent there is an issue of lack of communication and response or feedback from management. At this stage, the interpretant/signified or the sense made by the sign to the audiences starts to develop.

In addition to informing their friends and superiors of their problems, posting also gave them an emotional release. Everyone said they were disappointed and sad, at the same time, even when they were in the process of thinking of what to write in their posts. Facebook served as a conduit for their emotions, most especially employees who were let go. Other than the concerned employees, the reporter also said it was a way for him to show moral support and sympathy for his former colleagues. In connection to this, reactions to their posts gave them reassurance they are being heard by many people in the online space. Most of those who reacted either "liked" or reacted with "love" and "sad" to show emotional support.

However, checking on the profile of reactors, many were friends and families but mostly within their social circles. This is problematic since, based on their responses, they do not know if their sentiments can be heard by management or their superiors. One cameraman even went to the point of pessimism saying, "I do not care anymore because they do not listen to us in the first place." Suggesting that despite their efforts to be heard, their superiors turned "deaf" to their sentiments. This further bolster the thinking of being "muted" in their own organization.

Being present and engaged in social media means friends and other people in the online environment may copy or even share an original post. Since the image was posted and shared by many former employees for almost 1 week in Facebook meant that its use as an image of protest and resistance is effective. The use of the color bars has effectively come full circle since the aim of most of the respondents were to let the public and their company know about their sentiments. They did not expect other people to come into the picture, but it exceeded their expectations.

\section{Conclusion}

The utilization of the image "color bars" in the case of the retrenched TV5 employees presented a unique way of protesting in the online space. An image which is used behind-the-scenes in the TV industry was used for all the world to see, not in its original denotation, but in a repurposed meaning in social media specifically Facebook.

However, there is a problem on whether the color bars were seen in the much larger online sphere as a sign of protest. Firstly, the color bars were neutral in nature wherein the image was not normally associated as a symbol for emotions or even protest unlike explicitly saying it through text such as in placards in militant mobilizations. Even the texts (descriptions or captions) accompanying the image were generally vague and does not pose any hint of protest. At this point, the advocacy is already in a much-muted state resulting in frustration and dismay in the part of those affected.

Another complication is the reactors were only limited to their "closed" social circles namely their friends and families. The broader scope of people in Facebook do not see the post itself since a user cannot see a post of a certain user if they are not friends or are not shared outside of their social circles. Thus, much of those who understand the use of the color bars are only limited to those who also know and understand the work of the affected by the retrenchment.

And with television slowly losing its ground to online media, much of the audience or the general public especially in Facebook, do not see the significance of the color bars as a sign of protest. Those who posted the image tried to inform the more general audience however, the platform itself limited the reach of their advocacy. 
In a democratic country like the Philippines, it is an ironic situation wherein the media espouses and definitely enjoys freedom of expression, but their own retrenched and former employees have been oppressed in an implicit way even in online space. The internet may have given everyone access to freedom of expression, giving way for their voices to be heard. If, however, the oppressed are already in a certain state of conditioned oppression, the silence is still palpable in both image and text. Thus, the use of color bars in tandem with social media gave them a short stint onto breaking the silence but fell short of making their issues known to the general public.

\section{References}

Atkin, Albert, "Peirce's Theory of Signs", The Stanford Encyclopedia of Philosophy (Summer 2013 Edition), Edward N. Zalta (ed.), Date of Access: 3/1/2018. https://plato.stanford.edu/archives/sum2013/entries/peircesemiotics.

Bagheri, G, et.al., 2012. In Organization Silence: Basic Concepts and its Development Factors. Ideal Type of Management, Vol 1.No.1, Spring 2012. Date of Access: 3/1/2018. http://farabi.ut.ac.ir/old/stumags/ITM/ITM1_4.pdf.

Color Bars \& Test Patterns, Duke University Libraries. Date of access: 10/01/2018. https://blogs.library.duke.edu/bitstreams/2016/06/23/color-bars-test-patterns/

Dreyfus, H.,Rabinow, P., Foucault, M, 1983, Power and Truth. Beyond Structuralism and Hermeneutics, Second Edition. (Chicago:The University of Chicago Press), pp. 104-119.

Pinder, Craig and Harlos, Karen, 2001. Employee Silence: Quiescence and Acquiescenceas Responses to Perceived Injustice. Elsevier Science, Ltd. Date of Access: 3/2/2018. http://karenharlos.com/downloads/2017/06/pinder-harlos-2001-employee-silence.pdf

Rappler, TV5 lays off employees, Date of Access: 27/2/2017. https://www.rappler.com/nation/162707-tv5-layoffs-employees-last-batch 